\section{Avoiding TB's subversion}

\section{By Lev Osherovich, Senior Writer}

Mycobacterium tuberculosis has proven a tough bug to tame, with no effective vaccines on the market to stop the bacterium's spread. Now, a study published in the Proceedings of the National Academy of Sciences describes how M. tuberculosis evades peptide antigen presentation by dendritic cells (DCs), normally the first step in fighting an infectious agent. ${ }^{1}$

The researchers make a case for tuberculosis vaccines or adjuvants consisting of $M$. tuberculosis (Mtb) lipid antigens to enhance immunogenicity. As a next step, the team leader thinks efficacy experiments in non-human primates are needed to assess the overall relevance for human vaccination.

Meanwhile, a not-for-profit product development partnership focused on TB noted that the role of the immune system's other mechanisms must also be addressed to fully account for the bacterium's ability to evade immunity.

The only marketed vaccine for Mtb is the bacille Calmette-Guérin (BCG) vaccine. It consists of an attenuated strain of $M$. bovis, a close relative of $M$. tuberculosis. BCG protects infants from pulmonary tuberculosis, but doesn't protect adults or stop transmission or latent survival of the bug. BCG's mixed effectiveness is thought to result from, among other factors, a poor immune response to mycobacterial antigens. ${ }^{2}$

The PNAS study was led by Michael Brenner, chief of the division of rheumatology, immunology and allergy at Brigham and Women's Hospital, and addressed how Mtb subverts the activation of the acquired immune system by DCs.

Ordinarily, DCs prowl the skin and mucous surfaces, engulfing pathogens and packing them into endosomes. There, pathogen-derived peptides are processed into antigens for MHC class II (MHCII) presentation on the DC surface. The complex of MHCII and antigen then kickstarts acquired immunity by activating $\mathrm{T}$ cells in the lymph nodes.

Paradoxically, Mtb avoids this fate by hiding inside the endosomes of DCs and macrophages, the two favorite host cells for the bacterium. Brenner's team found that Mtb buys time inside DCs by sabotaging the presentation of peptide antigens by MHCII.

"Mtb is a very successful pathogen," said Brenner. "It's slower at releasing its protein antigens, giving it time to start growing to get a jump-start on the immune system."
However, a different antigen-presenting molecule called CD1 is impervious to subversion by Mtb, according to the study. A small minority of $\mathrm{T}$ cells can respond to lipids presented by $\mathrm{CD} 1$ molecules, launching the full range of cytotoxic and helper $\mathrm{T}$ cell responses against cells harboring the foreign lipids. ${ }^{3}$

"CD 1 is a second system that responds to lipid antigens, as opposed to proteins," said Brenner. "When Mtb infects dendritic cells, one system, MHCII, is compromised, but the other one, CD1, can still function."

\section{Slow peptides, fast lipids}

Brenner's team discovered the different immunogenic effects of Mtb peptides and lipids by infecting DCs with Mtb and testing their efficacy in activating cultured $\mathrm{T}$ cells that were specific for different types of antigen. Infected DC cells only weakly activated several T-cell lines specific for Mtb peptides, whereas $\mathrm{T}$ cells specific for Mtb lipids underwent rapid activation.

To uncover why Mtb lipids and peptides had such different effects, the team used confocal and electron microscopy to track the migration of MHCII and CD1 molecules within DCs following infection. Ordinarily, both MHCII and $\mathrm{CD} 1$ originate in the endosome, where they load up their antigens and then travel to the cell surface. This process triggers the maturation of DCs, which can be detected by morphological changes and the appearance of surface markers such as CD80 and CD83. ${ }^{4}$

Brenner's team found that Mtb triggered the premature maturation of MHCII, before antigens could be properly loaded. The team observed that MHCII rapidly moved from the endosome to the cell surface after infection, but in a nonfunctional state.

The reason may be that Mtb deliberately shuts down production of several of its own proteins when it enters the endosome, thus becoming invisible to the initial efflux of MHCII molecules. As a result, the T-cell response to peptide antigens is delayed until subsequent rounds of MHCII maturation several days later.

"MHCII-restricted cells aren't working because peptide antigen presentation is delayed," said Brenner. "Only after about three days do you get a peptide response."

Brenner's study "reveals one of the strategies of Mtb to escape immune responses," said François Romagne, CSO of Innate Pharma SA. The study shows that Mtb "fools the DCs by inducing maturation too rapidly, before they get time to be loaded by Mtb antigens," Romagne said.

Innate Pharma has a preclinical vaccine program focused on activation of $\gamma \delta \mathrm{T}$ cells, a subclass of $\mathrm{T}$ cells involved in innate immunity. $\gamma \delta \mathrm{T}$ cells "cross-talk very efficiently with DCs to increase their maturation and ability to present antigens," according to Romagne, and are thus good candidates for vaccines and adjuvants designed to outmaneuver immune evasion by Mtb. 


\section{TARGETS \& MECHANISMS}

\section{On the lipid side}

In contrast to MHCII, CD1 showed no Mtb-induced processing problems. After infection, CD1 remained in the endosome longer, clustering around the bacteria, where it presumably picked up lipid antigens. This more effective maturation process could explain why Mtb-derived lipids were better than peptides at stimulating T cells, according to Brenner.

None of the six Mtb vaccines in various stages of clinical testing specifically exploits CD1 lipid antigens to enhance immunity, however.

"It's not a very well known area of immunology to make vaccines using glycolipids," said Jerald Sadoff, president and CEO of Aeras Global TB Vaccine Foundation. "But this is a worthy place to look."

Lipids have previously been linked to both virulence and immunogenicity in Mtb. ${ }^{5,6}$ According to Sadoff, the CD1 mechanism described by Brenner could help explain the importance of lipids in the limited protection offered by BCG. He cited research showing that when certain lipids are lost from the BCG strain its efficacy decreases.

Aeras is a not-for-profit product development partnership funded primarily by the Bill \& Melinda Gates Foundation that works with private-sector vaccine makers to design and test Mtb vaccines.

The foundation's most advanced vaccine candidate is AERAS 485 (Oxford MVA85A). It is an attenuated vaccinia virus that expresses the Ag85 protein complex, which is made by Mtb during infection.

Sadoff told SciBX that AERAS 485 will enter Phase IIb trials in South Africa later this year, and will be "the first TB trial to go to Phase II in 85 years."

\section{Subunit vaccines}

According to Sadoff, current Mtb vaccine strategies involve either improving on BCG or introducing Mtb antigens through subunit vaccines that use heterologous expression systems such as adenovirus vectors.

He told SciBX that subunit vaccines now in the pipeline "are directed against secreted proteins that are made by the bug early in its life cycle."

One example, which Brenner's team looked at in the PNAS study, is Ag85. The team found that Ag85 is poorly immunogenic during actual Mtb infection.

However, Alexander von Gabian, CSO of Intercell AG, is not worried about the utility of subunit vaccines because when Mtb antigens are used in expression systems such as adenovirus, the whole bacterium isn't present to suppress immune recognition.

"This is a seminal paper, but at the same time I would not use this as evidence that a subunit vaccine won't work," said von Gabian.

Instead, he said the Brenner study shows that vaccinating with lipid antigens could be a good way to avoid escape of early peptide antigens from detection by the immune system.

Intercell makes the synthetic vaccine adjuvant IC31, a peptide and nucleic acid formulation that stimulates innate immune response through toll-like receptor 9 (TLR9). IC31 is a component of AERAS-404, a subunit vaccine in Phase I trials in partnership with AERAS, Statens Serum Institut (a research arm of the Danish Ministry of Health) and sanofi Pasteur, the vaccine division of sanofi-aventis Group.

\section{Wither lipids?}

The conclusions about the importance of lipid antigens may be more relevant to attenuated vaccines such as those based on BCG. But Sadoff cautioned that enhancing immunity by means of CD1 lipid antigens may only be a piece of the puzzle, because activation of CD1-specific T cells is only part of the immune system's set of defenses.

For example, Brenner's team did not address the effect of DCs on cytotoxic T cells, also known as $\mathrm{CD}^{+}$cells.

"There may be a way for the organism to partially escape the MHCII responses, but CD8 is also important" in acquired immunity to Mtb, Sadoff noted.

According to Brenner, the real test of usefulness of CD1-mediated immunity will be to immunize primates with $\mathrm{Mtb}$-derived lipid antigens. The usual mouse model of infection is difficult to use here because mice lack the appropriate CD1 subtype and must be engineered to express human CD1.

Brenner told SciBX that experiments are underway in mice transgenic for human CD1 to "vaccinate with a lipid, wait until it's gone and then come back with an infectious challenge to see if reactivation of innate immunity can quell infection."

Brigham and Women's Hospital holds several pending and granted patents on the use of CD1-mediated immunity for vaccination and therapy, but no specific patents were filed based on the work in this study, according to Brenner. The technology is available for licensing.

\section{REFERENCES}

1. Hava, D. et al. Proc. Natl. Acad. Sci. USA; published online August 6, 2008; doi:10.1073/pnas.0804681105

Contact: Michael Brenner, Brigham and Women's Hospital, Boston, Mass.

email: mmbrenner@rics.bwh.harvard.edu

2. Hoft, D.F. Lancet 372, 164-175 (2008)

3. Barral, D.C. \& Brenner, M.B. Nat. Rev. Immunol. 7, 929-941 (2007)

4. Reis e Sousa, C. Nat. Rev. Immunol. 6, 476-483 (2006)

5. Sirakova, T.D. et al. J. Bacteriol. 185, 2999-3008 (2003)

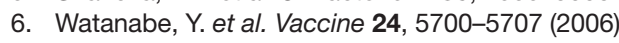

\section{COMPANIES \& INSTITUTIONS MENTIONED}

Aeras Global TB Vaccine Foundation, Bethesda, Md. Bill \& Melinda Gates Foundation, Seattle, Wash. Brigham and Women's Hospital, Boston, Mass. Innate Pharma SA (Euronext:IPH), Marseille, France Intercell AG (VSE:ICLL), Vienna, Austria sanofi-aventis Group (Euronext:SAN; NYSE:SNY), Paris, France Statens Serum Institut, Copenhagen, Denmark 